\title{
Toroidal universal drift instability: A global gyrokinetic study
}

\author{
J. Chowdhury, ${ }^{1}$ R. Ganesh, ${ }^{1, \text { a) }}$ S. Brunner, ${ }^{2}$ J. Vaclavik, ${ }^{2}$ and L. Villard ${ }^{2}$ \\ ${ }_{1}^{1}$ Institute for Plasma Research, Bhat, Gandhinagar 382428, India \\ ${ }^{2}$ CRPP, Association EURATOM-Confédération Suisse, EPFL, 1015 Lausanne, Switzerland
}

(Received 17 June 2010; accepted 27 August 2010; published online 19 October 2010)

\begin{abstract}
An electron density gradient driven instability identified as the toroidal branch of the universal drift instability is studied using a global gyrokinetic model treating both electrons and ions fully nonadiabatically and valid at all orders in the ratio of the Larmor radius to the wavelength. The physics of the magnetic drift resonance, Landau resonance and transit resonance, which are considered to be important for the toroidal universal mode, are kept for both species. A systematic parametric study is carried out for the mode. The toroidal universal drift mode is observed to sustain finite temperature gradient and can thus coexist with the temperature gradient driven modes and may contribute to the observed particle transport along with other drift modes. Especially at intermediate scales between the ion temperature gradient driven mode and electron temperature gradient driven mode, this branch of the drift instability can also be a plausible candidate for the observed particle loss. The effect of magnetic fluctuations on the mode is also investigated. In contrast to the slab mode, the toroidal branch of the universal drift mode is found to be strongly stabilized by electromagnetic effects at finite plasma $\beta$. Finally, the effect of trapped electrons on the universal mode is studied and compared with the other possible modes in the same parameter regime, namely, ion temperature gradient mode in the presence of trapped electrons and pure trapped electron modes. (C) 2010 American Institute of Physics. [doi:10.1063/1.3490238]
\end{abstract}

\section{INTRODUCTION}

In recent years, the tokamak has emerged as one of the most promising devices to realize controlled thermonuclear fusion as an alternate source of clean and sustainable energy. However, such a device suffers from various instabilities excited by the temperature and density gradients prevailing in the confined plasma. These instabilities are the origin of microturbulence leading to transport of heat and particles. Among the various instabilities, the ion temperature gradient (ITG) driven mode is considered to be the most deleterious mode for ion heat loss, while the trapped electron mode (TEM) and electron temperature gradient (ETG) driven modes are supposed to be the source of electron energy and particle loss from the device. In the absence of temperature gradients, there is another class of instabilities, which are driven even by the slightest density gradient in the plasma and are thus called universal drift instabilities. In the late 1980s, there had been a large effort to understand the basic physics of the universal drift instability. The motivation was that the universal drift mode was thought to be the dominant microinstability at that time and thus source of plasma transport in the various confining devices, such as, tandem mirror, tokamak, spheromak, stellarator, etc. In situations where the density profile is steeper than the temperature profile, one can indeed have fluctuations and associated transport dominated by the universal drift instability. Moreover, the universal instability is the simplest one in the class of drift waves and a thorough understanding of the mode can help get insight in the other modes of the family as well. The study of the universal drift wave had started with a very simple

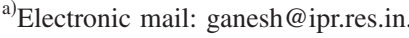

model, the shearless slab model, ${ }^{1,2}$ that revealed that the collisionless universal drift instability is always unstable in the presence of a density gradient and the absence of shear. The driving mechanism in this case is the wave particle resonance by the electrons coupled with the density gradients. The mode remains unstable, in the presence of weak but reasonable shear, when studied in the framework of the adiabatic electron model. Thus, up to that point, the speculation was that the universal mode without or with weak shear is always unstable. However, in contradiction to all previous works, Ross and Mahajan ${ }^{3}$ and Tsang et al. ${ }^{4}$ reported that the universal instability or collisionless drift instability in the presence of magnetic shear is always stable in the slab geometry, irrespective of the strength of the shear or transverse wave number when one takes into account the full electron dispersion function. The observed stability is attributed primarily to the stabilizing influence of the nonresonant electrons. These issues had further been addressed by Chen et $a .^{5}$ who concluded that the mode can be damped and marginally stable depending upon the strength of the shear and magnitude of the transverse wave number $k_{y}$. For example, in the case of sufficiently weak shear, i.e., with $L_{s} / L_{n}$ greater than a critical value (where $L_{s}$ and $L_{n}$ are, respectively, the measure of shear and density gradient scale length) collisionless drift waves are marginally stable at shorter wavelength or high wave number regime, where the electron dynamics usually play the pivotal role in determining the nature of an eigenmode. In the opposite limit, i.e., at longer wavelength or lower wave number regime where ion dynamics dominate, the eigenmode is damped. For strong shear, on the other hand, the eigenmodes are always stable irrespective of the wave number or wavelength. However, 
even in a sheared magnetic field, an absolute instability can be observed by the elimination of the stabilization produced by the off-resonant electrons by the turbulent diffusion near the mode rational surfaces. ${ }^{6}$ The investigation of the various properties of the universal drift instability in a more complex situation such as in the presence of finite toroidicity was undertaken by Cheng et al. ${ }^{7}$ Two eigenmodes were found to coexist that are brought about by the equilibrium variation along the field line. One mode is not localized in the ballooning coordinate and gets strongly stabilized due to the magnetic shear, much like the slab version of the mode. The other eigenmode is observed to have no slab counterpart. It is intrinsically driven by the toroidicity, partly localized, and weakly affected by the stabilization of the magnetic shear. It was shown that, while it is rendered as an absolute instability by the electron Landau resonance, ion Landau damping on the other hand has a stabilizing effect on the mode. It is important to note that the formulation in Ref. 7 uses the ballooning formalism applicable only in the high $n$ (toroidal mode number) limit, considers $k_{\perp} \rho_{L i} \ll 1$ and keeps the toroidal coupling effect only through the ion $\nabla B$ and curvature drift. The ion drift frequency, however, is assumed very small compared to the mode frequency $\omega$ and ion drift resonance is thus ignored. Studies involving more complex geometries, such as in small aspect ratio tokamaks or spheromaks ${ }^{8}$ were reported later. Modes are found to be less localized at different positions of poloidal angle when one removes the large aspect ratio assumption. However, except for the presence of more than one mode along the poloidal direction because of the strong equilibrium variations along the field line, the results are qualitatively the same as for the case of former large aspect ratio. The role of ion magnetic drift resonance and electron Landau resonance are discussed by Schep and Venema ${ }^{9}$ using the ballooning formalism with gyrokinetic theory. The conclusion is that along with magnetic drift resonance, one requires electron Landau resonance to make the mode unstable. However, this study precludes the role of the transit frequency of the thermal ions by assuming the mode frequencies to be higher than the transit frequency of the thermal ions. Berk and Domingues ${ }^{10}$ showed that the universal drift instability is stabilized by the presence of finite plasma $\beta$. This can be understood to result from the coupling of the universal drift wave to the Alfven mode and from the effect of ion Landau damping. Hastings and McCune, ${ }^{11}$ considering a slab geometry with finite $\nabla B$ and using gyrokinetic theory, find two mechanisms of stabilization of the mode by finite $\beta$ : (1) for small values of $\beta$ in the range $m_{e} / m_{i}<\beta \ll 1$, the stabilization is brought about by the coupling to the Alfven wave and (2) for $\beta \sim O(1)$, the stabilization is due to the compression of the perturbed plasma motion. The study of the universal drift instability thus evolved from a simple slab model to the toroidal geometry. Most of these formulations were, however, based on fluid or hybrid kinetic-fluid models. A few gyrokinetic models either exploited the ballooning formulation or a simple geometry. In this work we present a global, fully gyrokinetic study of the toroidicity driven universal drift mode using the numerical code EM-GLOGYSTO (Refs. 12-17) that considers both ions and electrons to be nonadiabatic. The formulation retains toroidal coupling effects due to both electron and ion $\nabla B$ and curvature drift with no assumption regarding the magnetic drift frequency of the ions and electrons compared to the mode frequency, thereby allowing full magnetic drift resonance by both species. The formulation also keeps the Landau damping term of both electrons and ions. Finite Larmor radius effects are kept up to all orders. More importantly, the present study retains the transit frequency resonance term in the nonadiabatic part of the density perturbation for both species as shown in Eqs. (1) and (2). It is to be noted that, we use a large aspect ratio, circular geometry for the tokamak, with no Shafranov shift. Though the universal toroidal mode is inherently due to the passing nonadiabatic electrons, effects of trapped electrons and trapped ions are also retained in the formulation. Furthermore, no collisional effect is considered in the formulation. With this model, various parametric studies of the toroidal branch of the universal mode have been carried out. We observe finite mode frequencies and growth rates beyond the critical $\eta=L_{n} / L_{T}$ for the ITG and ETG modes, where $L_{n}$ and $L_{T}$ are, respectively, the density and temperature gradient scale lengths. A comparative study of the contribution of the magnetic drift resonance as well as of the Landau resonance from both species toward the stability properties of the mode is performed by a systematic parametric scan. An electromagnetic study of the mode is also carried out that elucidates the effect of finite $\beta$ on the universal drift mode driven by toroidicity. The effect of trapped electrons on the universal mode is studied and growth rates and real frequencies are compared with the ITG mode and TEM.

The various parts of the paper are arranged as follows. Section II presents the basic set of gyrokinetic equations for the global formulation, both in the electrostatic and electromagnetic limit, Sec. III addresses the results of our numerical study using the global gyrokinetic formulation. Finally, Sec. IV presents a brief discussion of the obtained results.

\section{MODEL EQUATIONS}

In real space $\mathbf{r}$, for the species $j$, the perturbed density can be expressed as

$$
\begin{aligned}
\tilde{n}_{j}(\mathbf{r} ; \omega)= & -\left(\frac{q_{j} N}{T_{j}}\right)\left[\widetilde{\varphi}+\int d \mathbf{k} \exp (\iota \mathbf{k} \cdot \mathbf{r})\right. \\
& \left.\times \int d \mathbf{v} \frac{f_{M j}}{N}\left(\omega-\omega_{j}^{*}\right)\left(\mathcal{U}_{j}\right) \widetilde{\varphi}(\mathbf{k} ; \omega) J_{0}^{2}\left(x_{L j}\right)\right],
\end{aligned}
$$

where the first term on the right hand side corresponds to the adiabatic response, while the second term represents the nonadiabatic response of the particles to a perturbation with all its kinetic effects. Here, $q_{j}$ and $T_{j}$ stand, respectively, for the charge and temperature of species $j, N$ is the equilibrium density, $\omega_{j}^{*}=\omega_{n j}\left[1+\frac{\eta_{j}}{2}\left(\frac{v^{2}}{v_{\text {th }}^{2}}-3\right)\right]$ with the diamagnetic drift frequency $\omega_{n j}=\left(T_{j} \nabla_{n} \ln N k_{\theta}\right) /\left(q_{j} B\right)$, where $\nabla_{n}=-r B_{p} \frac{\partial}{\partial \psi}, \eta_{j}$ $=\left(d \ln T_{j}\right) /(d \ln N)$ is the ratio of the density to temperature gradient scale lengths, $v$ is the velocity amplitude, and $v_{t h j}$ is the thermal velocity of species $j . J_{0}\left(x_{L j}\right)$ is the Bessel function of argument $x_{L j}=k_{\perp} \rho_{L j}$, accounting for the finite Larmor radius effect to all orders. Note that here $(m, n)$ are poloidal 
TABLE I. Equilibrium profiles and parameters.

\begin{tabular}{lc}
\hline \hline \multicolumn{1}{c}{ Parameters: } & Equilibrium profiles: \\
\hline$\bullet B$ field: $B_{0}=1.0 \mathrm{~T}$ & $\bullet N$ and $T$ profiles \\
- Ion temperature: $T_{i 0}=T_{i}\left(s_{0}\right)=7.5 \mathrm{keV}$ & $N(s) / N_{0}=\exp \left[-\frac{a \delta s_{n}}{L_{n 0}} \tanh \left(\frac{s-s_{0}}{\delta s_{n}}\right)\right]$ \\
- Major radius: $R=2.0 \mathrm{~m}$ & $T_{i, e}(s) / T_{0}=\exp \left[-\frac{a \delta s_{T}}{L_{T}} \tanh \left(\frac{s-s_{0}}{\delta s_{T}}\right)\right]$ \\
- Minor radius: $a=0.5 \mathrm{~m}$ & $\delta s_{n}=0.35, \delta s_{T}=0.0$ \\
- Radius: $s=\rho / a 0.01<s<1.0, s_{0}=0.6$ & $\bullet q(s)=1.691+0.603 s^{2}+0.705 s^{4}$ \\
- $L_{n 0}=0.2 \mathrm{~m}, \eta_{i, e}\left(s_{0}\right)=0$ & such that $q\left(s=s_{0}\right)=2.0 ;$ \\
- $\tau(s)=T_{e}(s) / T_{i}(s)=3, \epsilon_{n}=L_{n 0} / R=0.1$. & Shear $\hat{s}$ is positive $\hat{s}=0.4$. \\
\hline \hline
\end{tabular}

and toroidal wave numbers, $q(s)$ is the safety factor, $k_{\theta}$ is the poloidal wavevector, $B_{p}$ is the poloidal magnetic field, and $i=\sqrt{-1}$. We consider a local Maxwellian for each species of mass $m_{j}$

$$
f_{M j}(\xi, \psi)=\frac{N(\psi)}{\left[\frac{2 \pi T_{j}(\psi)}{m_{j}}\right]^{3 / 2}} \exp \left[-\frac{\frac{1}{2} m v^{2}}{T_{j}(\psi)}\right] .
$$

Also, in Eq. (1) the term $\mathcal{U}_{j}$ represents the guiding center propagator for the passing particles. One can show that

$$
\mathcal{U}_{j}=\sum_{p, p^{\prime}} \frac{J_{p}\left(x_{t j}^{\sigma}\right) J_{p^{\prime}}\left(x_{t j}^{\sigma}\right)}{\omega-\sigma k_{\|}\left|v_{\|}\right|-p \omega_{t}} \exp \left[\iota\left(p-p^{\prime}\right)\left(\theta-\bar{\theta}_{\sigma}\right)\right]
$$

where $x_{t j}^{\sigma}=k_{\perp} \xi_{\sigma}, \quad \xi_{\sigma}=v_{d} / \omega_{t}, \quad \mu=\frac{v_{\perp}^{2}}{2 B}, v_{d}=\left(v_{\perp}^{2} / 2+v_{\Perp}^{2}\right) /\left(\omega_{c} R\right)$, $\omega_{t}=\sigma\left|v_{\|}\right| /(q(s) R), \quad \sigma= \pm 1 \quad\left(\operatorname{sign}\right.$ of $\left.v_{\|}\right), k_{\perp}=\sqrt{\kappa^{2}+k_{\theta}^{2}}, \quad k_{\|}$ $=[n q(s)-m] /(q(s) R)$, and $\bar{\theta}_{\sigma}$ is defined as $\tan \bar{\theta}_{\sigma}=-\kappa / k_{\theta}$ with $s=\rho / a, a$ and $R$ being the minor and major radius of a tokamak. Note that the $\nabla B$ and curvature drifts appear through the argument of Bessel functions $\left(x_{t j}^{\sigma}=k_{\perp} v_{d} / \omega_{t}\right)$ of Eq. (2). Hence in our model, Bessel functions in Eq. (2) bring about coupling between neighboring flux surfaces and also couple neighboring poloidal harmonics. Also, the argument of the Bessel functions $J_{p}$ in Eq. (2), $x_{t j}^{\sigma}=k_{\perp} \xi_{\sigma}$ depends on the transit frequency $\omega_{t}$ and can become of the order unity. Hence transit harmonic orders $p$ are to be chosen ac- cordingly. In this form, $\mathcal{U}_{j}$ contains effects such as transit harmonic resonances, parallel velocity resonances, and poloidal mode coupling. Introducing the quasineutrality condition

$$
\sum_{j} \tilde{n}_{j}(\mathbf{r} ; \omega)=0
$$

in the case of electrostatic fluctuations, one thus finally ends up with a generalized eigenvalue problem, where $\omega$ and $\tilde{\varphi}$ are the eigenvalue and the eigenvector, respectively. For a given toroidal mode number $n$ this can then be conveniently solved in the Fourier space $(\kappa, m)$ by Fourier decomposing the potential $\widetilde{\varphi}$ in Eq. (1) first and then taking the Fourier transform of $\tilde{n}_{j}$, to eventually obtain a convolution matrix in Fourier space. With the single charged (hydrogen) passing nonadiabatic ions and nonadiabatic electrons we have

$$
\sum_{\mathbf{k}^{\prime}} \sum_{j=i, e} \hat{\mathcal{M}}_{\mathbf{k}, \mathbf{k}^{\prime}}^{j} \widetilde{\varphi}_{\mathbf{k}^{\prime}}=0 .
$$

The considered axisymmetry of the system enables one in the linear analysis to fix the toroidal mode number $n$, so that the notation $\mathbf{k}=(\kappa, m)$ for the wave vector defines the radial wave number $\kappa$ and the poloidal wave number $m$. Thus, $\mathbf{k}=(\kappa, m)$ and $\mathbf{k}^{\prime}=\left(\boldsymbol{\kappa}^{\prime}, m^{\prime}\right)$. For the trapped electron and trapped ion formulation the reader is referred to Ref. 12.

For the electromagnetic case Eq. (1) for the perturbed density is modified as ${ }^{14}$
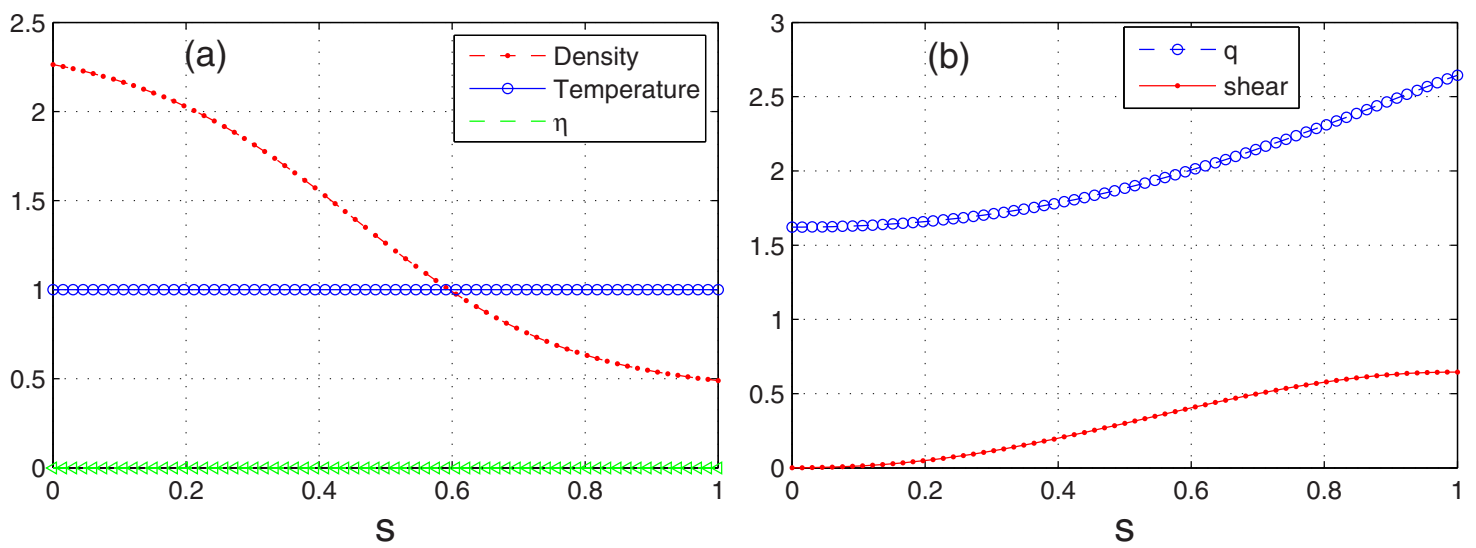

FIG. 1. (Color online) Equilibrium profiles to study the global toroidal universal drift instability mode (for parameters in Table I): (a) normalized density (dots), temperature (circle), $\eta_{i, e}$ (triangle), (b) safety factor $q$ (circle), and magnetic shear $\hat{s}$ (dots) profiles as functions of normalized radius $s=r / a$. Note that $q\left(s_{0}\right)=2.0, \hat{s}\left(s_{0}\right)=0.40, \epsilon_{n}\left(s_{0}\right)=0.1$, and $\tau\left(s_{0}\right)=3.0$ for $s_{0}=0.6$. 

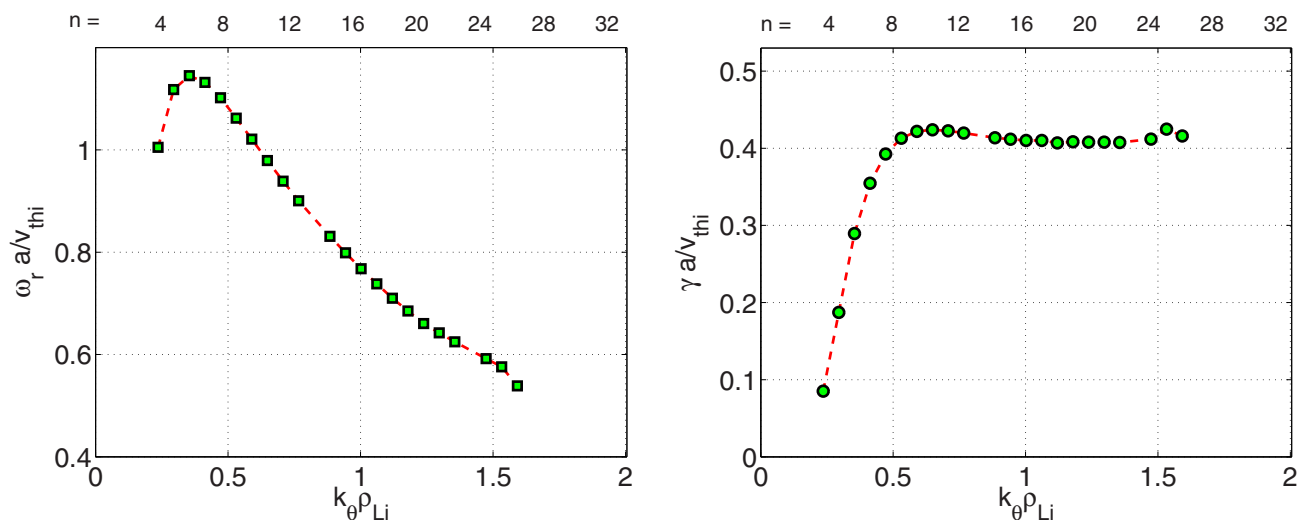

FIG. 2. (Color online) Real frequency and growth rate for the electrostatic case corresponding to the parameters in Table I and profiles shown in Fig. 1.

$$
\begin{aligned}
\widetilde{n}_{j}(\mathbf{r} ; \omega)= & -\left(\frac{q_{j} N}{T_{j}}\right)\left[\tilde{\varphi}+\int d \mathbf{k} \exp (\mathbf{k} \cdot \mathbf{r}) \int d \mathbf{v} \frac{f_{M j}}{N}\left(\omega-\omega_{j}^{*}\right)\right. \\
& \left.\times\left(\mathcal{U}_{j}\right)\left[\widetilde{\varphi}(\mathbf{k} ; \omega)-v_{\|} \widetilde{A}_{\|}(\mathbf{k} ; \omega)\right] J_{0}^{2}\left(x_{L j}\right)\right]
\end{aligned}
$$

where $\widetilde{A}_{\|}$is the component parallel to the equilibrium magnetic field of the vector potential associated to the perturbation. In addition to $\widetilde{n}_{j}$, one has to consider the fluctuation of the parallel current density given by

$$
\begin{aligned}
\widetilde{j}_{\| j}(\mathbf{r} ; \omega)= & -\left(\frac{q_{j}^{2}}{T_{j}}\right)\left[\int d \mathbf{k} \exp (\iota \mathbf{k} \cdot \mathbf{r}) \int v_{\|} d \mathbf{v} f_{M j}\left(\omega-\omega_{j}^{*}\right)\right. \\
& \left.\times\left(\mathcal{U}_{j}\right)\left[\widetilde{\varphi}(\mathbf{k} ;)-v_{\|} \widetilde{A_{\|}}(\mathbf{k} ;)\right] J_{0}^{2}\left(x_{L j}\right)\right] .
\end{aligned}
$$

Along with the quasineutrality condition Eq. (3), Ampere's law

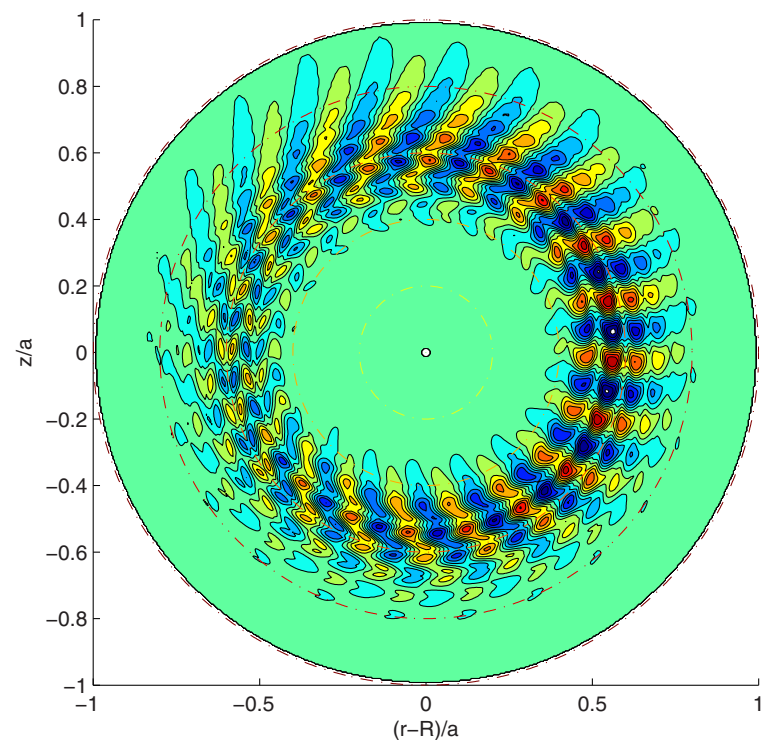

$$
\frac{1}{\mu_{0}} \nabla_{\perp}^{2} \widetilde{A}_{\|}=-\sum_{j} \widetilde{j_{\| j}}
$$

will finally close the set of equations, to give a linear system of equations of the form

$$
\sum_{\mathbf{k}^{\prime}} \sum_{j=i, e} \hat{\mathcal{M}}_{\mathbf{k}, \mathbf{k}^{\prime}}^{j}\left(\begin{array}{l}
\widetilde{\varphi}_{\mathbf{k}^{\prime}} \\
\tilde{A}_{\| \mathbf{k}^{\prime}}
\end{array}\right)=0 .
$$

Simple diagnostics for various physical quantities are computed as averages over the eigenmode. For example mode-averaged $k_{\theta}^{2}$ for the electrostatic case is computed as

$$
\left\langle k_{\theta}^{2}\right\rangle=\frac{\int d \rho \Sigma_{m}\left|\frac{m}{\rho} \varphi_{(\rho, m)}\right|^{2}}{\int d \rho \Sigma_{m}\left|\varphi_{(\rho, m)}\right|^{2}} .
$$

The above shown averaging procedure is suitably extended to the electromagnetic cases by including $\widetilde{A}_{\|}$mode structure averaging as follows:

FIG. 3. (Color online) (left) The electrostatic mode structure for toroidal mode number $n=10, k_{\theta} \rho_{L i}=0.58$ corresponding to the parameters in Table I and profiles shown in Fig. 1. (right) Poloidal component of $\widetilde{\phi}$ in (top) radial Fourier representation and (bottom) radial direct space. 


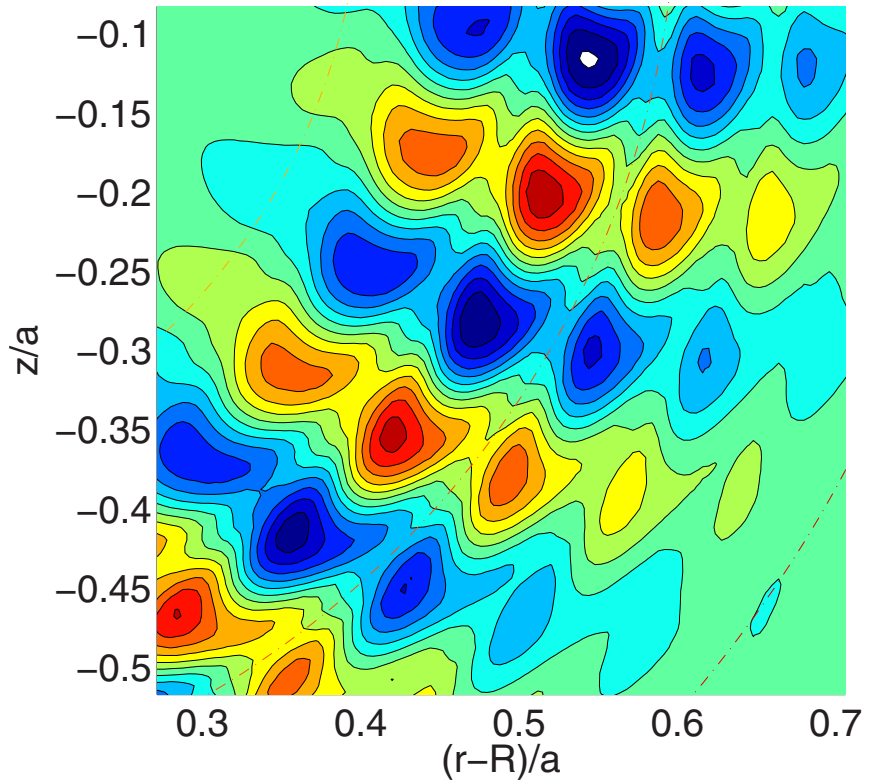

FIG. 4. (Color online) A closeup view of the mode structure shown in Fig. 3.

$$
\left\langle k_{\theta}^{2}\right\rangle=\frac{\int d \rho \Sigma_{m}\left|\frac{m}{\rho} \varphi_{(\rho, m)}\right|^{2}+\int d \rho \Sigma_{m}\left|\frac{m}{\rho} A_{\|_{(\rho, m)}}\right|^{2}}{\int d \rho \Sigma_{m}\left|\varphi_{(\rho, m)}\right|^{2}+\int d \rho \Sigma_{m}\left|A_{\|_{(\rho, m)}}\right|^{2}} .
$$

\section{RESULTS AND DISCUSSION}

In the present section, we will delineate the results from the global linear gyrokinetic numerical analysis. It is to be noted that the mode frequencies and growth rates are expressed in units of $v_{t h i} / a$ throughout the paper.

\section{A. Profiles and parameters}

Let us consider the following profiles and parameters for a plasma with hydrogen ions (see Table I).

The equilibrium profiles corresponding to these parameters are shown in Fig. 1. Note that results obtained in the subsections from $B$ to $J$ are without the effect of trapped electrons.

\section{B. Growth rate $\gamma$ and real frequency $\omega_{r}$ versus $k_{\theta} \rho_{L i}$}

The dispersion diagram for the toroidal universal drift instability with real frequency and growth rate plotted versus the normalized poloidal wave number $k_{\theta} \rho_{L i}$ is shown in Fig. 2. The upper axis presents the corresponding toroidal mode numbers. The real frequency is in the direction of the electron diamagnetic drift frequency. The real frequency at very low $k_{\theta} \rho_{L i}$ increases first and then peaks at the value of $k_{\theta} \rho_{L i}=0.4$ corresponding to $n \simeq 6$. After this point, it starts falling with $k_{\theta} \rho_{L i}$ in a monotonic way. The growth rate, on the other hand, initially increases until the point $k_{\theta} \rho_{L i} \simeq 0.58$ corresponding to the toroidal mode number $n \simeq 10$ and is practically constant at larger value of $k_{\theta} \rho_{L i}$. It is apparent from this observation that the toroidal branch of the universal drift instability spans from the low wave number or
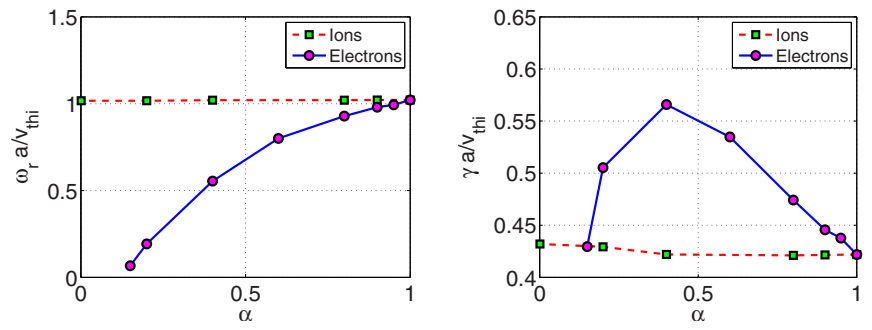

FIG. 5. (Color online) The effect of electron and ion Landau resonance for the mode $n=10$ corresponding to $k_{\theta} \rho_{L i}=0.58$. This is done by weighting the Landau resonance term by $\alpha$ and running it from 0 to 1 .

longer wavelength regime, where ion dynamics are dominant, to higher wave number or shorter wavelength regime, where usually electron dynamics play the dominant role. This is in contrast to the observation in the slab case, ${ }^{5}$ which is marginally stable at high wave number regime and damped in the low wave number regime.

\section{Electrostatic mode structure}

In the present section, we discuss the global electrostatic mode structure of the toroidal branch of the universal drift mode. Figure 3 displays the potential contours on a poloidal cross section of the tokamak in the left panel for $k_{\theta} \rho_{L i}=0.58$ corresponding to the toroidal mode number $n=10$. The various poloidal components of the potential with coupling brought about by the toroidicity, both in Fourier and real space, are presented in the right panel for $k_{\theta} \rho_{L i}=0.58$ corresponding to the toroidal mode number $n=10$. A few important issues to be noted in this context are: (1) The mode structure is quite global passing through many mode rational surfaces. (2) It exhibits a weak ballooning character, with a finite amplitude observed at the favorable curvature side (high field side). In the right panel, coupling of poloidal components has been shown across the minor radius, with a maximum amplitude at $s=\rho / a=0.6$, where the density gradient peaks. The points in the upper axis, labeled by the corresponding poloidal mode numbers $m$ display the position of the mode rational surfaces where $k_{\|}(m, n)=0$. Corresponding to each of these points, one can see a dip in the potential corresponding to each poloidal mode number. These dips correspond to $k_{\|}(m, n)=0$ surfaces where $\left|\omega / k_{\|}\right| \gg v_{\text {the }}, v_{\text {the }}$ being the electron thermal velocity. Thus, the strong effect of the off-resonant electrons is clearly visible from this figure. The convergence in the Fourier space for the considered mode is presented in the upper part of the right panel of Fig. 3. Figure 4 presents a closeup view of the mode considered here.

\section{Effect of Landau resonance}

To investigate the effect of the Landau resonance of electrons and ions on the toroidal universal drift instability, one can artificially put a multiplying factor, say $\alpha$, in front of the $k_{\|} v_{\|}$term in the denominator of the propagator for both species [see Eq. (2)] and decrease it gradually from 1 to 0 , once for ions, keeping full electron Landau resonance effect, and vice versa. It is to be noted that only the values 0 and 

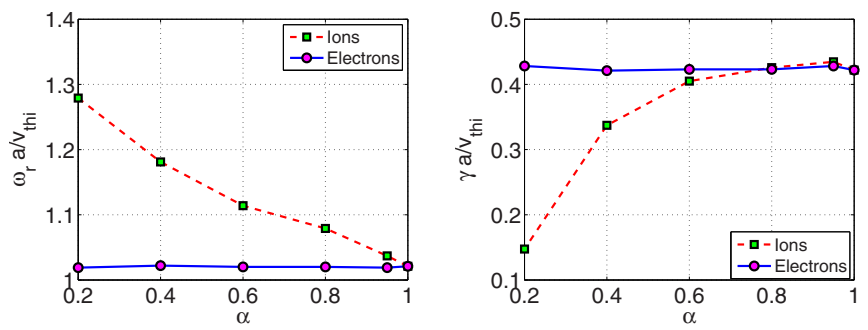

FIG. 6. (Color online) Effect of ion and electron magnetic drift resonance for the mode $n=10$ corresponding to $k_{\theta} \rho_{L i}=0.58$. This is done by weighting the magnetic drift term by $\alpha$ and running it from 0 to 1 .

1 of the artificial factor $\alpha$ are physically meaningful. The other values of $\alpha$ simply represent a fractional weight to the Landau resonance term in the propagator so as to enable us to track the importance of the Landau effect continuously. Thus the value 1 will refer to the case of full Landau resonance term taken into account and 0 the complete omission of the Landau resonance term from the propagator. It is clear from Fig. 5 that the ion Landau resonance apparently has no significant effect on the growth rate as well as on the mode frequency compared to that of the electrons. For the electron Landau resonance, the growth rate exhibits a nonmonotonic dependence on the electron Landau resonance weighting parameter $\alpha$. For example, at lower values of $\alpha$ the growth rate increases and again falls at higher values. Regarding the mode frequency, in contrast to the growth rate, it increases monotonically with $\alpha$ for the electrons, while it has little variation in the case of ions. Thus, with the complete omission of the electron Landau resonance, the mode may become nonexistent even if one keeps the other destabilizing factors intact.

\section{E. Effect of magnetic drift resonance}

In toroidal geometry, a mode will certainly have magnetic drift resonance if its frequency is of the same order as the magnetic drift frequency. We have looked at the effect of magnetic drift resonance for both species on the toroidal universal mode. This is done in a similar way as for the study of the previous section, by putting a multiplying factor $\alpha$ in front of the magnetic drift resonance term, $x_{t j}$ appearing as arguments of the Bessel functions in the numerator of the propagator in Eq. (2). To be noted again that, while doing the scan for one species, the full weight $\alpha=1$ is kept for the other species. One notes a destabilizing effect due to the magnetic drift resonance of ions when the multiplying factor $\alpha$ increases. As shown in Fig. 6, the real frequency decreases with the increasing multiplying factor $\alpha$, while the growth rate increases and starts saturating as one approaches $\alpha=1$, i.e., for the full magnetic drift resonance term in the ion propagator. Electron magnetic drift resonance however does not play a significant role for the universal drift instability, as is clear from Fig. 6.

\section{F. Shear scan}

Though magnetic shear has a strong stabilizing influence on the universal drift mode, in a slab model, the toroidal resonance effects, intrinsic to toroidal geometry, can reduce the shear damping of the mode. The shear scan is displayed in Fig. 7. It is clear that for the parameters chosen in this study, the critical value of shear, beyond which the mode gets stabilized, is of the order of 1 . The growth rate and real frequency, however, do not decay monotonically with increasing shear. The growth rate rather increases weakly at low shear and then starts decreasing with increasing shear. Similarly the real frequency of the mode also decays with increasing shear. The stabilization of the mode by shear in the presence of finite toroidicity can be understood as follows. The shear damping of the universal drift mode in the slab geometry is basically due to the convection of energy away from the mode rational surfaces. In the presence of finite toroidicity, the toroidal coupling effect inhibits this convection of wave energy, and thus reduces the shear damping. ${ }^{7}$ Similar effects of toroidicity on shear induced stabilization was also observed for high- $n$ toroidal universal drift instabilities.

\section{G. Toroidicity scan}

The real frequency and growth rate of the mode versus $\epsilon_{n}=L_{n} / R$ are presented in Fig. 8 . The toroidicity scan is done by varying $R$, but keeping $R q, L_{n}, n q$, and $a$ constant. While the real frequency decreases almost monotonically, the growth rate, on the other hand increases first with toroidicity, peaks at around $\epsilon_{n} \simeq 0.1$, and then starts falling for larger

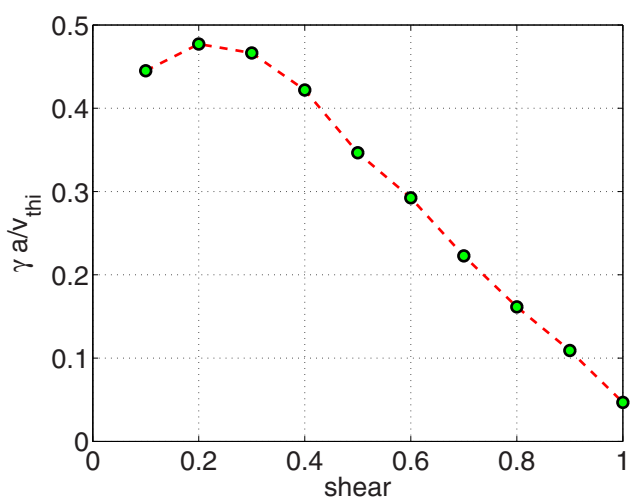

FIG. 7. (Color online) Shear scan for the mode $n=10$ corresponding to $k_{\theta} \rho_{L i}=0.58$ at position $s=s 0=0.6$, where the density gradient peaks. For these scans the safety factor at $s=s_{0}$ is kept at the fixed value $q=2.0$. 

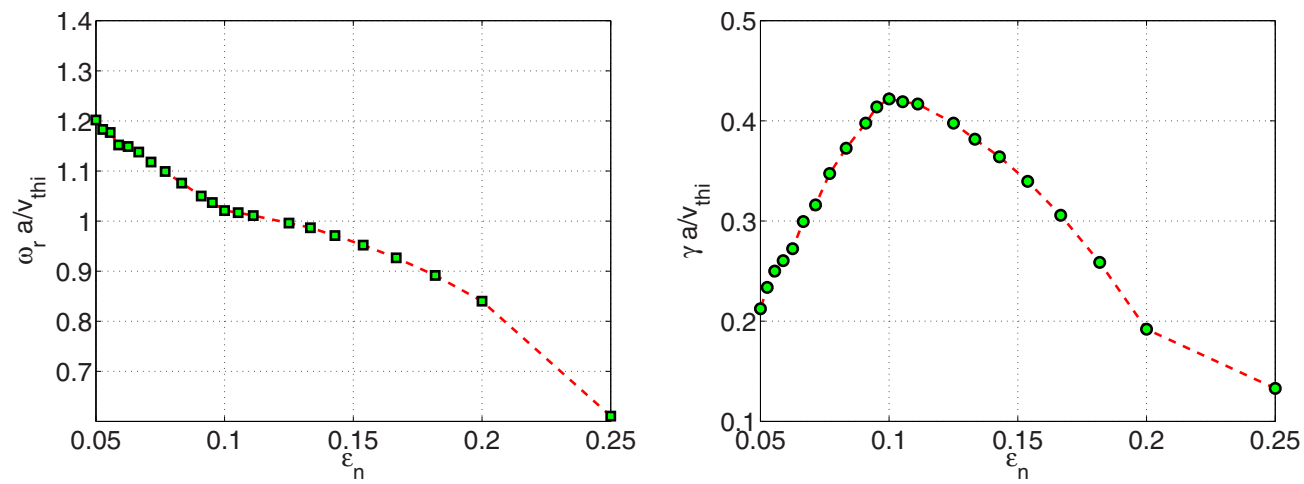

FIG. 8. (Color online) Real frequency and growth rate for $\epsilon_{n}=L_{n} / R$ for the parameters and profiles as in the Table I and Fig. 1 in the case of mode $n=10$ corresponding to $k_{\theta} \rho_{L i}=0.58$. Note that $a, L_{n}, R q$, and $n q$ are kept constant in this scan.

values of $\epsilon_{n}$. Since, $\epsilon_{n} \rightarrow 0$ implies $R \rightarrow \infty$, i.e., the cylindrical limit, the toroidal driving term becomes weak at low $\epsilon_{n}$. Since the mode is basically driven by the magnetic drift resonance, the growth rate increases with $\epsilon_{n}$ for low values. However, for large enough values of $\epsilon_{n}$ the mode becomes off-resonant with respect to the magnetic drift frequency, which increases with $\epsilon_{n}$ and growth rate falls down. Also, increasing $\epsilon_{n}$ implies decreasing $R$, which means reduced connection length $\sim R q$ between the favorable and unfavorable magnetic field. This nonmonotonic dependency of growth rate on $\epsilon_{n}$ for toroidal universal drift modes has not been reported before.

\section{H. $\tau=T_{e} / T_{i}$ scan}

The dependence of the mode frequency and growth rate for the toroidal universal drift instability on the temperature of the species is elucidated in the present section. Figure 9 displays the plots of the mode frequency and the growth rate corresponding to $k_{\theta} \rho_{L i}=0.58(n=10)$ as a function of the ratio of temperatures of electrons and ions, i.e., $T_{e} / T_{i}=\tau$. To be noted that in this scan the ion temperature $T_{i}$ is kept constant, while varying only the electron temperature $T_{e}$. The real frequency increases monotonically with the magnitude of $\tau$, i.e., with increasing electron temperature. The growth, on the other hand, exhibits a nonmonotonic character: increases at first with $\tau$, peaks at around $\tau=5.0$, and then starts decaying with increasing $\tau$. One may correlate this result with the role of electron Landau resonance on the universal mode, as the electron distribution in the vicinity of parallel resonant velocity which is strongly dependent on the thermal velocity and so the temperature of the electrons is the key factor in determining Landau damping or inverse Landau damping of the mode.

\section{Effect of temperature gradient}

Thus far, the entire analysis has been carried out considering flat temperature profiles, that is, zero temperature gradients by putting $\delta s_{T}=0$ in the profiles displayed in Table I. In the present section, we incorporate profile variation to the temperatures of both the ions and electrons. This is achieved by using a finite value for $\delta s_{T}$, which is chosen as 0.2 in this case. Since most tokamaks contain temperature gradients in the pressure profile, it is thus necessary to look at the effect of the temperature gradient on the toroidal universal drift instability. This is done by evaluating the real frequency and growth rate against $\eta_{i, e}=L_{n} / L_{T}$, keeping $L_{n}$ constant and varying $L_{T}$. Three cases are considered here: (1) the temperature gradient scale lengths for both electrons and ions are increased simultaneously, (2) the temperature gradient of only ions is increased, keeping that of the electrons zero, and (3) the temperature gradient of only electrons is increased, keeping that of the ions zero. The last two options may be relevant to experimental situations with preferential ion heating [e.g., ion cyclotron resonance heating (ICRH)] or elec-

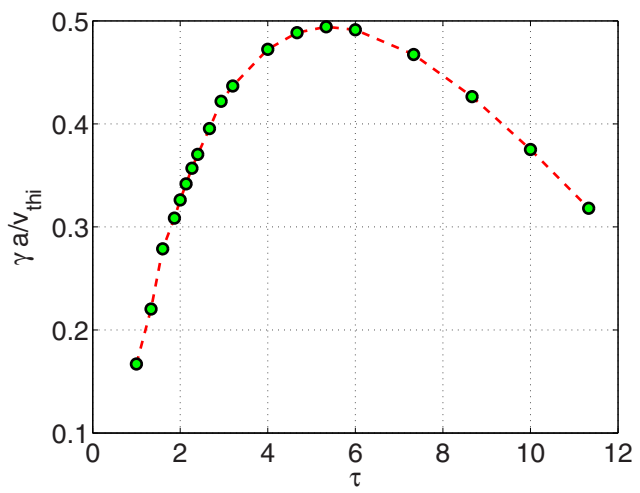

FIG. 9. (Color online) Real frequency and growth rate for $\tau=T_{e} / T_{i}$ and for the parameters and profiles of Table I and Fig. 1 in the case of the mode $n=10$ corresponding to $k_{\theta} \rho_{L i}=0.58$. 

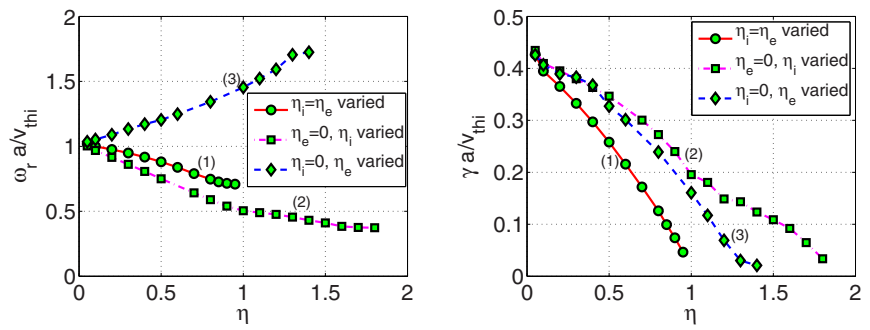

FIG. 10. (Color online) Real frequency and growth rate in the case of a temperature gradient scan for the parameters and profiles of Table I and Fig. 1 for the mode $n=10$ corresponding to $k_{\theta} \rho_{L i}=0.58$. The flat temperature profile in Table I has been replaced with $\delta s_{T}=0.2$ instead of 0 for the previous cases.

tron heating [e.g., electron cyclotron resonance heating $(\mathrm{ECRH})]$, respectively. The results for all the cases are presented in Fig. 10. The real frequency is reduced with increasing temperature gradient for the cases 1 and 2, while it increases in the case 3 . The growth rate, on the other hand, decreases in all three cases. However, it decays more slowly when the electron temperature profile is flat, as apparent from case 2. It is clear from case 3 that the mode exhibits a finite growth rate for values of $\eta_{e}$ beyond the critical value for ETG instability $\left(\eta_{e} \simeq 1.0\right)$. Thus, the point to be noted is that, even in the presence of finite $\eta$ above the critical value for the temperature gradient driven modes to get destabilized, the universal drift instability preserves finite growth rate. It is observed from case 2 that the ITG has weaker effect on the mode. It has finite growth rate even after the critical value of $\eta_{i}$ for the ITG driven mode $\left(\eta_{i} \simeq 1.0\right)$. Thus, one may conclude that, in some situations as delineated in the last two cases, temperature gradient driven modes and the universal drift mode driven by the density gradient can coexist. One other important point to be noted here is that the toroidal universal drift mode is unstable in the $\tau$ domain from 1 to more than 10, as evident from Fig. 9, while ETG modes are stable at higher values of $\tau$ even with finite $\eta_{e}$. So electron transport at high $k_{\perp} \rho_{L i}>1$ with larger values of $\tau$ may have contributions from the toroidal universal drift mode as well. Similarly, at low $k_{\perp} \rho_{L i}$, where ITG is dominant, the electron transport can be due to this mode, as it appears to be unstable in regions where ITG is pronounced.

\section{J. Electromagnetic effects}

\section{1. $k_{\theta} \rho_{L i} \operatorname{scan}$}

The electrostatic assumption is justified in a low $\beta$ plasma. However, considering the higher $\beta$ environment in the present day devices, it is of interest to study the effect of the electromagnetic fluctuation on the toroidal branch of the universal mode. In this section, we perform a $k_{\theta} \rho_{L i}$ scan for the growth rate and mode frequency in the presence of transverse magnetic perturbations with $\beta=0.001$ in the zero temperature gradient limit. The corresponding results are plotted in Fig. 11, with the upper axis representing the respective toroidal mode numbers $n$. For the purpose of comparison, we also plot the purely electrostatic values for the real frequency and growth rate of the mode (same results as in Fig. 2). At very low $k_{\theta} \rho_{L i}$, the real frequency increases first and
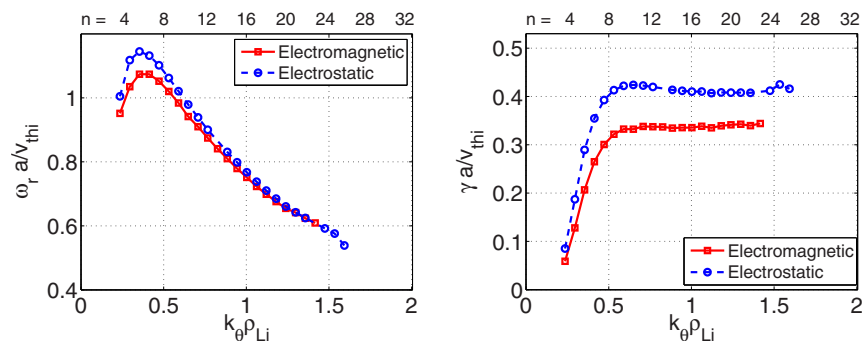

FIG. 11. (Color online) Real frequency and growth rate for the electrostatic (dashed curve) and electromagnetic case (solid curve) for the parameters in Table I and profiles as shown in Fig. 1. The value of $\beta$ considered here is 0.001 .

then peaks at a value of $k_{\theta} \rho_{L i} \simeq 0.4$ corresponding to $n=6$. Beyond this point, the frequency starts falling monotonically with $k_{\theta} \rho_{L i}$ (or $\left.n\right)$. The growth rate however increases until $k_{\theta} \rho_{L i} \simeq 0.58$ (or $n=10$ ) and then becomes practically constant. It is apparent from the figure for the real frequency that the effect of finite $\beta$ is more pronounced at lower $k_{\theta} \rho_{L i}$, and the real frequency is reduced in this region. Going toward the higher $k_{\theta} \rho_{L i}$ the effect of $\beta$ seems to be weaker on the mode frequency. The growth rate, on the other hand, is substantially reduced by finite plasma $\beta$. A $\beta$ of value 0.001 brings almost $20 \%$ reduction in the growth rate as compared to the electrostatic case. A complete $\beta$ scan is presented in Sec. III J 2 clearly illustrating the stabilizing effect of $\beta$.

A global mode structure for the electromagnetic case for $n=10$ and $\beta=0.001$ corresponding to $k_{\theta} \rho_{L i} \simeq 0.58$ is shown in Figs. 12 and 13. The electrostatic part $\widetilde{\phi}$ (Fig. 12) is very similar to the purely electrostatic mode in Fig. 3. The $\widetilde{A}_{\|}$ component (Fig. 13), on the contrary, apparently shows a weak antiballooning character, being weaker at the outboard side than the inboard side. The convergence in the radial and poloidal Fourier space for the mode has been depicted in the upper part of the right panel in Fig. 13. The lower panel presents the radial dependence of various poloidal mode numbers $m$. It retains the effect of nonresonant electrons at $k_{\|}(m, n)=0$ surfaces. The antiballooning character of the $\widetilde{A}_{\|}$ mode structure and the stabilization of the mode in the presence of finite $\beta$ are all related to the inherent electrostatic nature of the toroidal universal drift instability.

\section{2. $\beta$ scan}

A complete $\beta$ scan for the mode with $k_{\theta} \rho_{L i}=0.58$ $(n=10)$ is displayed in Fig. 14. Both the real frequency and growth rate are reduced with increasing $\beta$. The complete stabilization occurs at $\beta \simeq 1.1 \%$. This is in contrast to earlier investigations in slab geometry, where the value of critical $\beta$ was much higher. ${ }^{11}$ The observed stabilization is perhaps due to the coupling of the wave with the Alfven perturbation.

The relative strength of the electromagnetic to the electrostatic character is shown in Fig. 15, measured as the ratio of flux surface averaged squared $\widetilde{A}_{\|}$to $\widetilde{\phi}$ with increasing value of $\beta$ expressed in percentage. It is clear from this plot that the strength of the magnetic fluctuation in comparison to the electrostatic fluctuation increases almost linearly with increasing plasma $\beta$. 

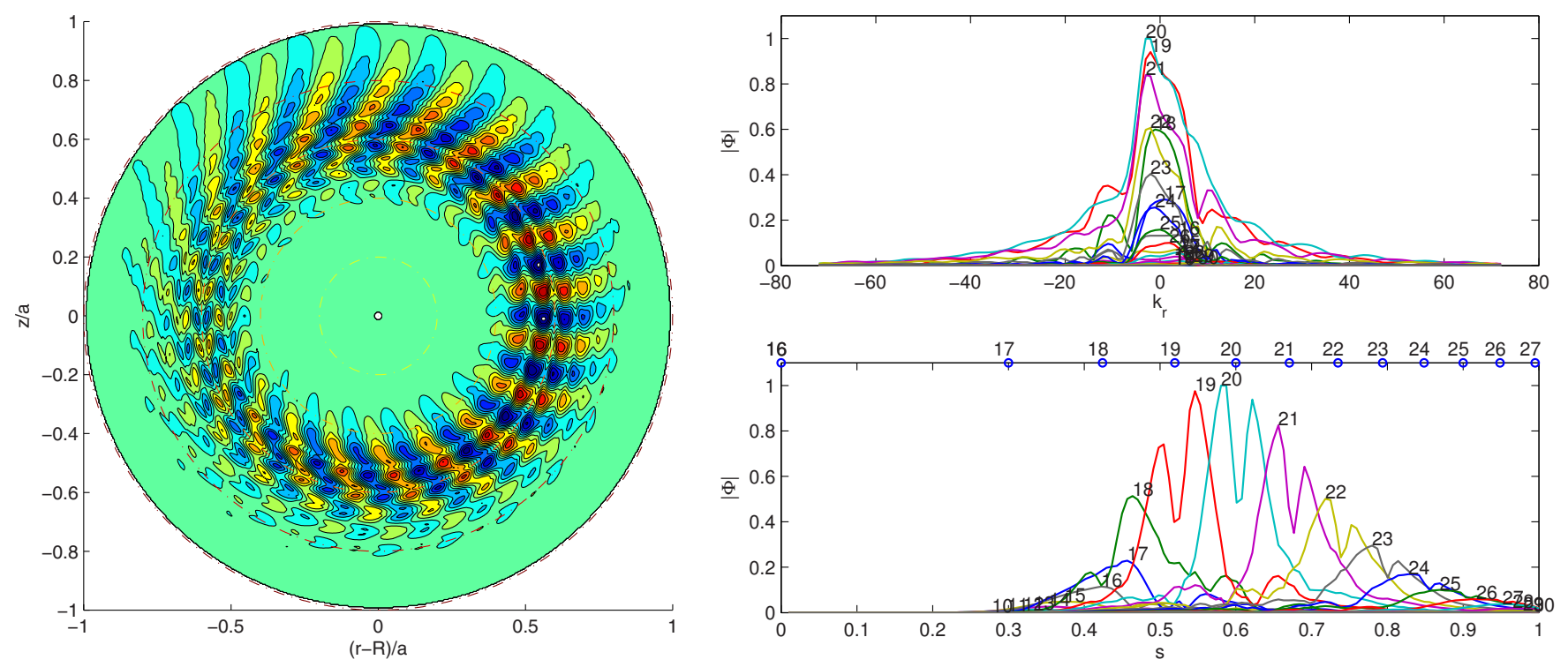

FIG. 12. (Color online) (left) The global mode structure for the $\widetilde{\phi}$ component in the poloidal cross section in the electromagnetic case for $n=10$, $k_{\theta} \rho_{L i}=0.58$, and $\beta=0.001$. (right) Poloidal component of $\widetilde{\phi}$ in (top) radial Fourier representation and (bottom) radial direct space.
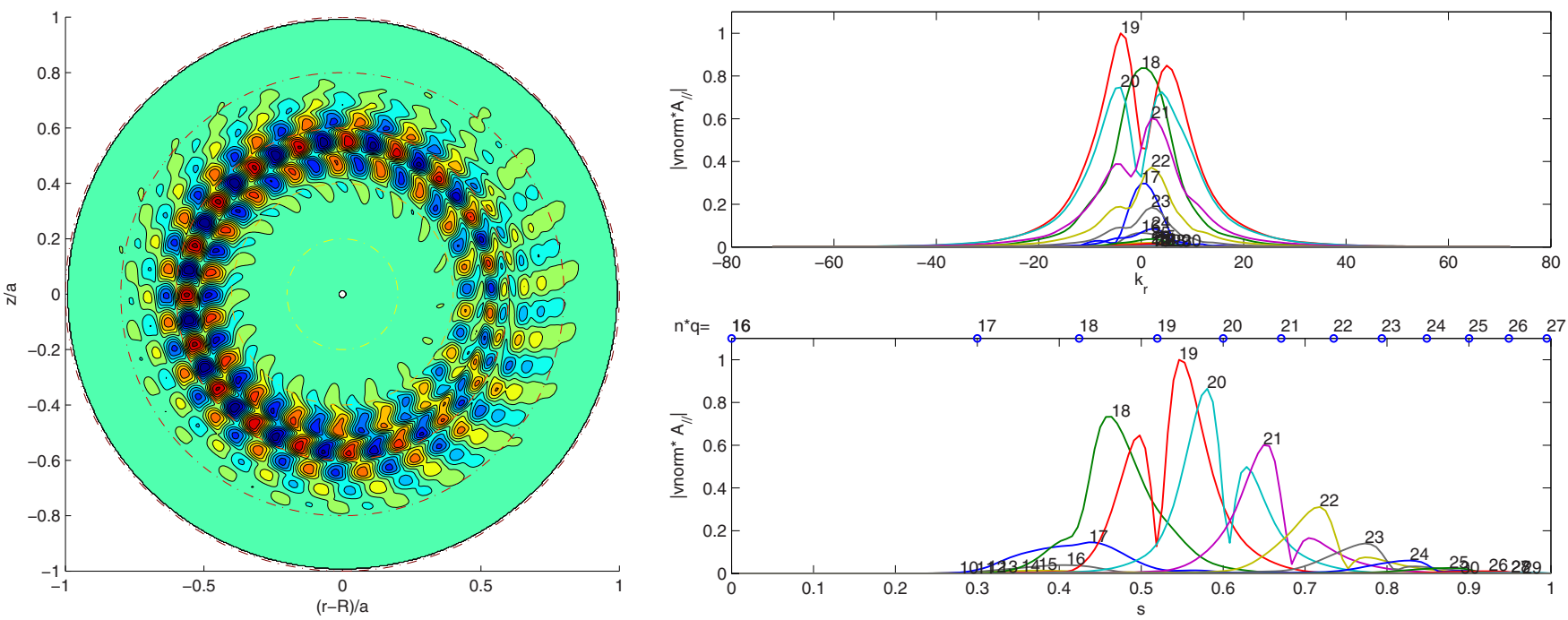

FIG. 13. (Color online) (left) The global mode structure for the $\widetilde{A}_{\|}$component in the poloidal cross section in the electromagnetic case for $n=10$, $k_{\theta} \rho_{L i}=0.58$, and $\beta=0.001$. (right) Poloidal component of $\widetilde{\phi}$ in (top) radial Fourier representation and (bottom) radial direct space.
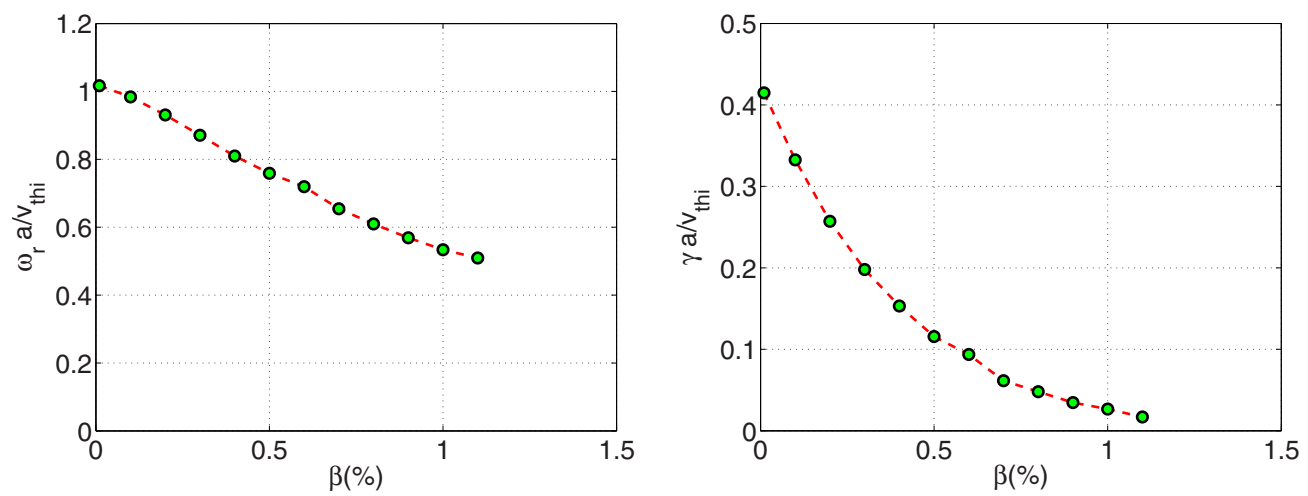

FIG. 14. (Color online) $\beta$ scan for the mode frequency and growth rate for the parameters and profiles as in Table I and Fig. 1 for the mode $n=10$ corresponding to $k_{\theta} \rho_{L i}=0.58$. 


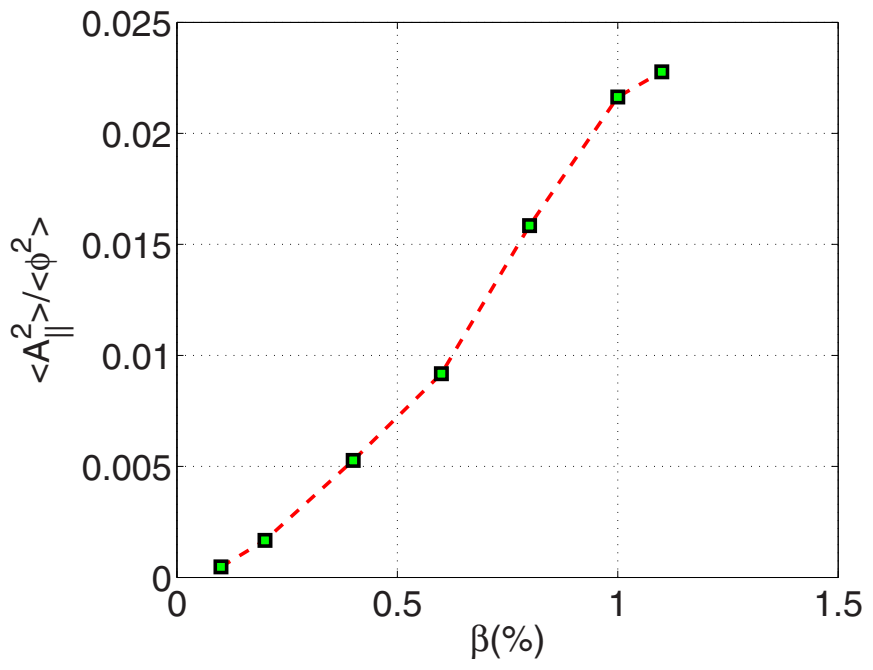

FIG. 15. (Color online) Electromagnetic ratio with increasing function of $\beta$ for the parameters and profiles as in Table I and Fig. 1 for the mode $n=10$ corresponding to $k_{\theta} \rho_{L i}=0.58$.

\section{K. Effect of trapped electron}

As clearly demonstrated by the above results, the global toroidal universal drift instability is triggered by purely passing electron dynamics. However, as a toroidal device is bound to have some fraction of trapped electrons, it would be interesting to obtain the effect of trapped electrons on the purely universal drift mode studied in the previous sections. To this end, an extensive $\eta$ scan is performed with and without trapped electrons for the electrostatic case. To identify the most unstable mode, the other drift instabilities such as ITG with trapped electrons as well as the TEM branches are computed together with the universal mode. The combined data are plotted in Fig. 16. There are several interesting points to be noted: (1) The trapped electron coupled universal drift mode and pure universal drift mode have distinct real frequencies and growth rates. (2) In contrast to the pure universal drift mode studied in previous sections, whose growth rate was shown to decrease with increasing $\eta$, the trapped electron coupled universal drift mode appears to be more unstable with increasing $\eta$. This study indicates that in the presence of trapped electrons, the nature of the universal drift mode is predominantly "trapped electron like." (3) To make a better quantitative comparison, growth rates and real frequencies of the pure TEM, the ITG mode with trapped electrons (ITG-TE) for the same equilibrium parameters are also plotted. For the parameters studied here, it appears that the trapped electron coupled universal drift modes in the presence of $\eta \geq 1$ have growth rates comparable to ITG-TE or TEMs and could contribute substantially to the overall transport.

\section{CONCLUSIONS}

In the present work we performed a global linear gyrokinetic study of the toroidal universal drift mode driven by the density gradient in the presence of finite toroidicity on the intermediate scale $k_{\perp} \rho_{L i}$. The model considers both passing electrons and ions to be fully nonadiabatic, incorporating toroidal coupling effects, magnetic drift resonances, Landau resonance effects, transit harmonic resonances, finite Larmor radius to all orders, and orbit width effect for both species. The effect of finite $\beta$ is also studied in the frame of an electromagnetic model that retains the transverse magnetic perturbation. However, effects of collisions and Shafranov shift have been dropped. Furthermore, the model considers large aspect ratio circular cross section for the tokamak plasma. The major results are as follows:

- The growth rate increases at lower $k_{\theta} \rho_{L i}$ until $k_{\theta} \rho_{L i} \simeq 0.58$ and starts saturating thereafter. The real frequency too increases at lower $k_{\theta} \rho_{L i}$ and then decays monotonically with $k_{\theta} \rho_{L i}$ at larger $k_{\theta} \rho_{L i}$.

- The electrostatic mode structure is global and exhibiting structure at mode rational surfaces.

- Studying the effect of Landau resonance for both electrons and ions shows weak dependence of the frequency and growth rate on ion Landau damping and a strong dependence on electron Landau damping preserving finite growth rate in both cases.

- Both electron and ion magnetic drift resonance terms are considered in the formulation. The ion magnetic drift resonance does play a significant role in making the toroidal
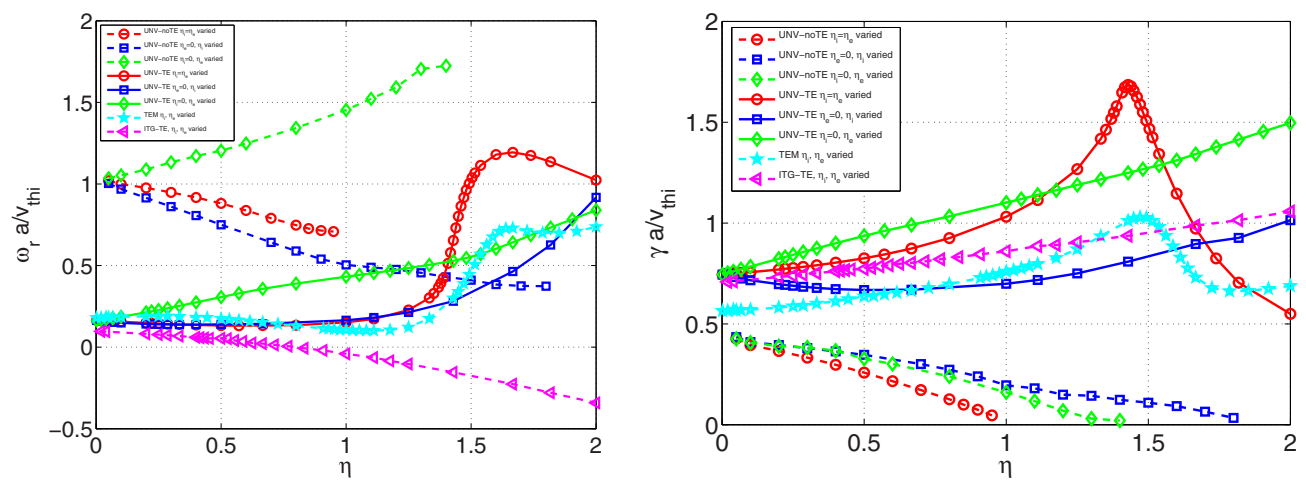

FIG. 16. (Color online) Real frequency and growth rate vs temperature gradient for different unstable modes in the presence of trapped electrons in the same regime defined by the parameters and profiles of Table I and Fig. 1 for the mode $n=10$ corresponding to $k_{\theta} \rho_{L i}=0.58$. The flat temperature profile in Table I has been replaced with $\delta s_{T}=0.2$ instead of 0 for the previous cases. The three dashed curves (circle, square, diamond) are for universal mode without trapped electrons (same as Fig. 10), three solid lines (circle, square, diamond) are for universal mode in the presence of trapped electrons (UNV-TE), the dashed curve with triangles is for ITG mode with trapped electrons (ITG-TE), and the solid curve with stars is for pure TEM. 
branch of the universal drift mode unstable, while electron magnetic drift resonance has a weak effect on the stability property of the mode.

- As reported in earlier works for high $n$ modes $\left(k_{\theta} \rho_{L i} \gg 1\right)$, the toroidicity driven universal drift mode is found to be stable beyond a shear value $\hat{s} \simeq 1$, even for low $n$ modes.

- The nonmonotonic dependency of the growth rate on $\epsilon_{n}=L_{n} / R$ in a toroidicity scan (varying $R$ and keeping $L_{n}$, $a, R q$ and $n q$ constant) is demonstrated here for the first time.

- The mode is unstable in a fairly large domain of $\tau=T_{e} / T_{i}$ ranging from 1 to more than 10 , thus clearly showing that in regions of $\tau$ where the ETG mode is believed to be stable, electron transport can be due to this toroidal universal drift mode.

- The $\eta$ scan for both ions and electrons shows that the universal drift mode driven by toroidicity can coexist with the temperature gradient driven modes. Therefore electron transport at low $k_{\theta} \rho_{L i}$ may have contributions from the mode under investigation. Similarly at higher $k_{\theta} \rho_{L i}$, where ETG is thought to be the main driving mechanism for electron transport, this mode may also contribute.

- The electromagnetic effect is found to be strongly stabilizing in the present case. The $\tilde{A}_{\|}$component of the mode structure exhibits antiballooning character. The mode gets stabilized at $\beta \simeq 1.1 \%$. The relative magnetic fluctuation amplitude $\left\langle\widetilde{A}_{\|}^{2}\right\rangle /\left\langle\widetilde{\phi}^{2}\right\rangle$ varies almost linearly with the magnitude of $\beta$.

- Trapped electrons enhance the growth rate of the universal mode. However, the universal mode changes its character regarding its dependence on the density and temperature gradients. While in the absence of trapped electrons the universal mode decays with the temperature gradient, trapped electrons, on the contrary, enhances the growth rate of the mode. The universal mode with trapped electrons exhibits, qualitatively, the same character as the TEM. Also, it has a comparable growth rate to the trapped electron coupled ITG mode in the parameter range considered in this study.

\section{ACKNOWLEDGMENTS}

Authors (J.C. and R.G.) are thankful to IPR Computer Centre for its support during the course of this work. The computations were performed on a 34-node Xeon cluster with fast-ethernet interconnect at IPR.

${ }^{1}$ L. D. Pearlstein and H. L. Berk, Phys. Rev. Lett. 23, 220 (1969).

${ }^{2}$ N. T. Gladd and W. Horton, Jr., Phys. Fluids 16, 879 (1973).

${ }^{3}$ D. W. Ross and S. M. Mahajan, Phys. Rev. Lett. 40, 324 (1978).

${ }^{4}$ K. T. Tsang, P. J. Catto, J. C. Whitson, and J. Smith, Phys. Rev. Lett. 40, 327 (1978).

${ }^{5}$ L. Chen, P. N. Guzdar, R. B. White, P. K. Kaw, and C. Oberman, Phys. Rev. Lett. 41, 649 (1978).

${ }^{6}$ S. P. Hirshman and K. Molvig, Phys. Rev. Lett. 42, 648 (1979).

${ }^{7}$ C. Z. Cheng and L. Chen, Phys. Fluids 23, 1770 (1980).

${ }^{8}$ R. Marchand and P. N. Guzdar, Phys. Fluids 24, 2183 (1981).

${ }^{9}$ T. J. Schep and M. Venema, Plasma Phys. Controlled Fusion 27, 653 (1985).

${ }^{10}$ H. L. Berk and R. R. Domingues, J. Plasma Phys. 18, 31 (1977).

${ }^{11}$ D. E. Hastings and J. E. McCune, Phys. Fluids 25, 509 (1982).

${ }^{12}$ S. Brunner, M. Fivaz, T. M. Tran, and J. Vaclavik, Phys. Plasmas 5, 3929 (1998).

${ }^{13}$ G. L. Falchetto, J. Vaclavik, and L. Villard, Phys. Plasmas 10, 1424 (2003).

${ }^{14}$ R. Ganesh, P. Angelino, J. Vaclavik, and L. Villard, Phys. Plasmas 11, 3106 (2004).

${ }^{15}$ R. Ganesh and J. Vaclavik, Phys. Rev. Lett. 94, 145002 (2005).

${ }^{16}$ J. Chowdhury, R. Ganesh, P. Angelino, J. Vaclavik, L. Villard, and S. Brunner, Phys. Plasmas 15, 072117 (2008).

${ }^{17}$ J. Chowdhury, R. Ganesh, S. Brunner, J. Vaclavik, L. Villard, and P. Angelino, Phys. Plasmas 16, 052507 (2009). 